\title{
Estrogen Administration Attenuates Immobilization-Induced Skeletal Muscle Atrophy in Male Rats
}

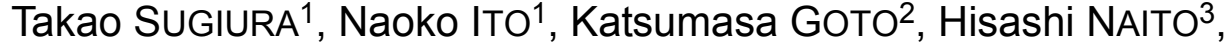 \\ Toshitada YOSHIOKA ${ }^{4,5}$, and Scott K. POWERS 6 \\ ${ }^{1}$ Department of Exercise and Health Sciences, Faculty of Education, Yamaguchi University, Yamaguchi, 753-8513 Japan; ${ }^{2}$ Faculty of \\ Rehabilitation, Toyohashi Sozo University, Ushikawa, Toyohashi 440-8511, Japan; ${ }^{3}$ Department of Exercise Physiology, School of Health \\ and Sports Science, Juntendo University, Inba, Chiba, 270-1695 Japan; ${ }^{4 H}$ irosaki Gakuin University, Hirosaki, Aomori 036-8577, Japan; \\ ${ }^{5}$ Department of Physiology, St. Marianna University School of Medicine, Miyamae, Kawasaki City, Kanagawa, 216-8511 Japan; and \\ ${ }^{6}$ Department of Applied Physiology and Kinesiology, Center for Exercise Science, University of Florida, Gainesville, Florida 32611, USA
}

\begin{abstract}
We tested the hypothesis that estrogen administration would retard immobilization-induced muscle atrophy in adult male rats. The rats were injected for 24 days with either estrogen $\left(40 \mu \mathrm{g} / \mathrm{kg}^{-1}, \beta\right.$-estradiol 3-benzoate in olive oil vehicle), or vehicle alone. At day 14 of estrogen treatment, the hindlimb muscles of one leg were immobilized in plantar flexion position by the use of a plaster cast. Following 10 days of immobilzation, the atrophic and the contralateral soleus muscles were both removed and analyzed to determine the level of muscle atrophy along with the measurement of the protein levels of $\mathrm{Cu}$-Zn-superoxide
\end{abstract}

dismutase (Cu-Zn-SOD), heat shock protein 72 (HSP72), and selected proteases. Compared to placebo animals, estrogen treatment significantly reduced (-35\%) muscle atrophy. Further, estrogen significantly abridged the expression of the calcium-activated protease, calpain, in the atrophied hindlimb muscle. In contrast, estrogen treatment did not alter the protein levels of HSP72 in the immobilized soleus muscle. These results support the postulate that estrogen attenuates the rate of disuse muscle atrophy, partly because of reductions in immobilization-induced calcium-activated protease levels.

Key words: estrogen, atrophy, oxidative stress, protease.

$\mathrm{I}_{\mathrm{t}}$ It is well known that reduced contractile activity in skeletal muscle results in a rapid onset of muscle atrophy because of decreased protein synthesis and increased proteolysis [1]. The disuse muscle atrophy can occur in a variety of clinical situations that require prolonged bed rest and/or limb immobilization. The loss of muscle mass from inactivity in special populations (such as the elderly and small children) is of particular importance given that extensive muscle atrophy can result in a loss of mobility and an inability to perform the activities of daily living. Therefore the development of countermeasures to prevent disuse muscle atrophy is important.

Theoretically, countermeasures that increase muscle protein synthesis and/or decrease muscle protein breakdown will retard the rate of muscle atrophy during prolonged periods of inactivity. In that regard, investigators have used several approaches with varying degrees of success [1]. A unique and promising countermeasure to retard disuse muscle atrophy is the delivery of antioxidants to the muscle to prevent oxidative stress that occurs during periods of inactivity. Several lines of evidence suggest that disuse-induced oxidative stress in skeletal muscle contributes to muscle atrophy $[1,2-4]$. The first evidence that oxidants contributed to disuse muscle atrophy was provided by Kondo and colleagues [2]. They showed that the concentrations of thiobarbituric acid-reacting substances (TBARS) and oxidized glutathione and the activities of copper-zinc-superoxide dismutase (Cu-Zn-SOD) and glutathione reductase increased in atrophied muscle induced by immobilization. Thus this work revealed that the immobilization of skeletal muscles was associated with oxidative injury in the muscle, and that this form of muscle atrophy could be further retarded by the delivery of vitamin E, a lipid soluble antioxidant [2]. Indeed, immobilization induces an increase of $\mathrm{Ca}^{2+}$-dependent cysteine proteinase (calpain) activity [5]. The increase of calpain activity induces concomitantly with the increase in lysosomal proteinases activities, cathepsin B and cathepsin L, in the unweighted rat soleus muscle [6].

Although the mechanism responsible for antioxidant protection against muscle atrophy remains debatable, growing evidence suggests that the prevention of disusemediated oxidative stress protects against disuse muscle atrophy by diminishing protease activity and reducing the rate of proteolysis $[1,4]$. Note, however, that while selected antioxidants can reduce disuse muscle atrophy, not all

Received on Jun 15, 2006; accepted on Oct 18, 2006; released online on Oct 21, 2006; doi:10.2170/physiolsci.RP006906 Correspondence should be addressed to: Takao Sugiura. Department of Exercise and Health Sciences, Faculty of Education, Yamaguchi University, Yamaguchi 753-8513, Japan. E-mail: takahito@yamaguchi-u.ac.jp 


\section{T. SUGIURA et al.}

antioxidant interventions are capable of retarding it [7]. Therefore the search for a highly effective countermeasure against muscle atrophy remains an active area of research.

In regard to the prevention of disuse muscle atrophy, a unique and potentially effective countermeasure against muscle atrophy is the female steroid hormone, estrogen. Indeed, estrogen has been shown to play a role in protection against membrane damage resulting from a variety of cellular stresses. In reference to estrogen and skeletal muscles, it has been widely reported that estrogen can reduce contraction-induced skeletal muscle damage [8-14]. For example, TBARS in females during a 30-day training period was lower than in males [10]. The expression of heat stress protein 72 (HSP72), which functions as molecular chaperones and plays a role in maintaining cellular homeostasis and protecting cells and tissues during periods of stress was significantly low in estradiol-administred male rats compared with control male rats following exercise [12]. Thus estrogen is an effective cellular antioxidant and can provide membrane stability by protecting muscle fibers against oxidative stress-induced lipid peroxidation.

Therefore because of evidence that estrogen can act as a cellular antioxidant, we formulated the postulate that estrogen would protect against disuse muscle atrophy. So the current study tested the hypothesis that the administration of estrogen to male rats would diminish skeletal muscle atrophy induced by immobilization.

\section{MATERIALS AND METHODS}

Animals. These experiments followed the guiding principles for the care and use of animals in the field of physiological sciences (The Physiological Society of Japan) and were approved by the Yamaguchi University animal use committee. Male Wistar strain rats (14 weeks of age, $n$ $=12$ ) were randomly assigned into two groups: (i) vehicle-treated and (ii) estrogen-treated. Throughout the experiments, the rats were individually housed in a climatecontrolled room $\left(26 \pm 1{ }^{\circ} \mathrm{C}, 56 \pm 1 \%\right.$ relative humidity, and $12 \mathrm{~h}: 12 \mathrm{~h}$ light-dark photo period) and fed standard rat chow and water ad libitum. The animals received daily doses of either $40 \mu \mathrm{g} / \mathrm{kg}$ body mass $\beta$-estradiol 3-benzoate (Sigma, St. Louis, Mo., USA) in virgin olive oil or virgin olive oil (vehicle) alone. This level of estrogen has been shown to increase estrogen levels in rodent skeletal muscle [13]. Injections were made subcutaneously into the neck fold and were administered for 24 consecutive days. Following 14 days of injections, one hindlimb was immobilized for 10 days in plantar flexion with plaster (Castlight, ALCARE Co., Ltd., Japan). At the completion of the experiment, the rats were sacrificed by an overdose of sodium pentobarbital $(100 \mathrm{mg} / \mathrm{kg})$. After the animals reached a surgical plane of anesthesia, the soleus muscles of both legs were quickly removed, carefully weighed, and then rapidly frozen in liquid nitrogen. The muscles were stored at $-80^{\circ} \mathrm{C}$ until they were analyzed.

Because of the small mass of tissue available in the atrophied soleus muscle, our measurements were limited to essential proteins required to test our proposed hypothesis. Specifically, we determined the protein levels of $\mathrm{Cu}-$ Zn-SOD, HSP 72, calpain, and cathepsin L in the soleus muscles of both control and immobilized limbs.

Muscle preparation. Muscle samples were minced and homogenized in ice-cold homogenization buffer $(10 \mathrm{mM}$ Tri-HCl, pH 7.6, $10 \mathrm{mM} \mathrm{NaCl}$, 0.1 mM EDTA). Homogenates were centrifuged at $3,000 \times g$ for $30 \mathrm{~min}$ at $4^{\circ} \mathrm{C}$, and the protein concentration of the supernatants was determined in triplicate (BioRad, Richmond, CA, USA).

Measurement of muscle protein content. Noncollaogenous soleus muscle protein was measured using techniques described by Sugita et al. [17]. In each experimental condition, the total protein of the immobilized muscle was compared to muscle from the control limb and expressed as a percent.

SDS-PAGE, Western blotting, and immunodetection. Muscle samples were solubilized in sample loading buffer (30\% glycerol, $5 \%$-mercaptoethanol, 2.3\% SDS, 62.5 $\mathrm{mM}$ Tris-HCl, $\mathrm{pH} 6.8,0.05 \%$ bromophenol blue) at a concentration of $1.5 \mathrm{mg} / \mathrm{ml}$ and incubated at $60^{\circ} \mathrm{C}$ for $10 \mathrm{~min}$. Proteins were then separated by $12.5 \%$ SDS-PAGE and transferred onto a polyvinylidene difluoride membrane (Millipore, Bedford, MA, USA), using a Bio-Rad minitrans-blot cell (Bio-Rad Laboratories, Richmond, CA, USA ) at a constant voltage of $100 \mathrm{~V}\left(1 \mathrm{~h}\right.$ at $\left.4^{\circ} \mathrm{C}\right)$ in transfer buffer (25 mM Tris-HCl, pH 8.3, $192 \mathrm{mM}$ glycine and $20 \%$ methanol) [18]. After protein transfer, the membranes were then blocked for $1 \mathrm{~h}$ at room temperature using a blocking buffer [5\% nonfat dry milk in Tween Tris buffer saline (T-TBS; $20 \mathrm{mM}$ Tris-HCl, $150 \mathrm{mM} \mathrm{NaCl}$, $0.05 \%$ Tween-20, $\mathrm{pH} 7.5)]$. Following serial washing with T-TBS, the membranes were incubated with primary antibodies to HSP72 (diluted 1:10,000 in T-TBS, SPA-812; StressGen, Victoria, B.C., Canada), Cu-Zn-SOD (diluted 1:10,000 in T-TBS, SOD-101, StressGen, Victoria, B.C., Canada), Cathepsin L (diluted 1:100 in T-TBS, SC-6499, Santa Cruz Biotechnology, Santa Cruz, CA, USA) and calpain subunit ( $\mu$ - or $\mathrm{m}$-Calpain, diluted 1:1,000 in TTBS, MAB3083, Chemicon International, Temecula, CA., USA) primary antibodies overnight at $4^{\circ} \mathrm{C}$. After several washes in T-TBS, the membranes were incubated with either anti-rabbit (A-3682, Sigma, St. Louis, MO,USA) or anti-mouse (A-4312, Sigma) alkaline phospatase conjugated secondary antibodies (diluted 1:30,000 in T-TBS) for 1 hour at room temperature. The membranes were then washed three times in TTBS for $5 \mathrm{~min}$. The protein bands were visualized by the use of an Alkaline Phosphatase Conjugate Substrate Kit (Bio-Rad). The quantification of each detected protein was performed using computerized 
image analysis, and the protein content was expressed as the relative ratio (\%) to the non-immobilized muscles of the placebo-treated group.

Statistics. Body weight comparisons between groups were made using an unpaired Student's $t$-test. All other group comparisons were made using a two-way analysis of variance for multiple comparisons followed by a Tukey's post hoc test. A $P$ value of $<0.05$ was considered significant. All data are reported as means \pm SD.

\section{RESULTS}

Body weights in the estrogen-treated animals were significantly lower than in the placebo group (Fig. 1A). Note, however, that the estrogen treatment did not alter soleus muscle weights in the non-immobilized limb, indicating that the body weight differences between the experimental groups were not due to altered muscle weights (Fig. 1B). In the placebo group, the atrophied soleus weights were significantly lower than the contralateral muscles. Although soleus muscle weights in the estrogen-treated group tended to be lower in the immobilized muscles compared to the contralateral muscles, these differences did not reach significance (Fig. 1B).

The soleus weight-to-body weight ratio in immobilized limbs was significantly lower compared to contralateral limbs in both the placebo and estrogen treated groups (Fig. 2A). Note, however, that the magnitude of soleus

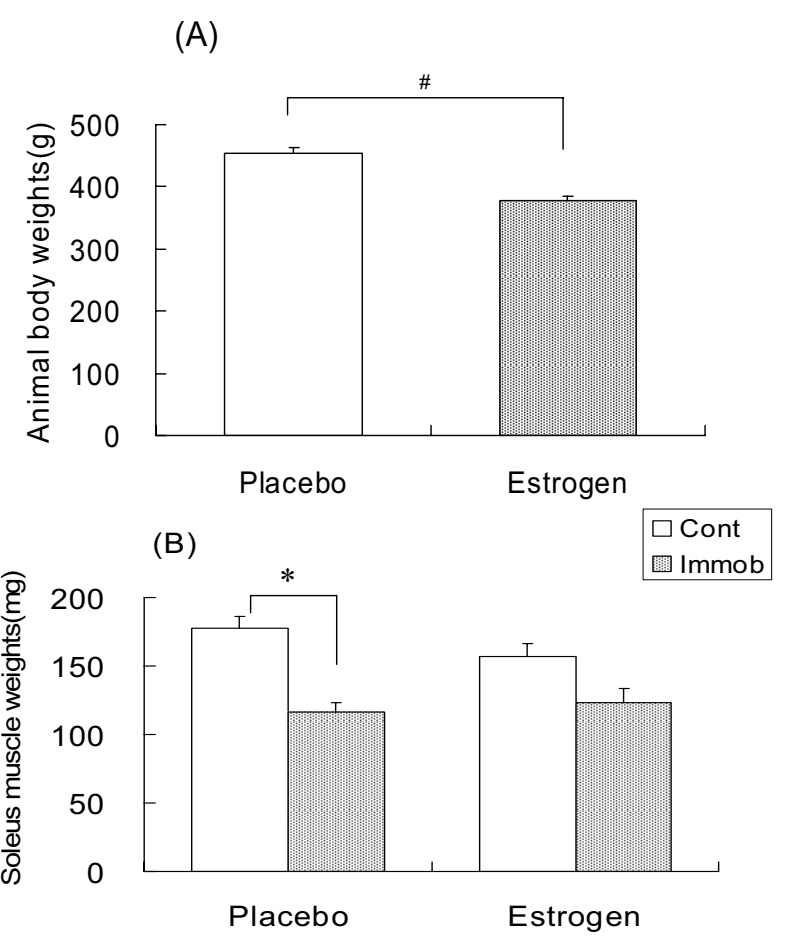

Fig. 1. Effects of estrogen treatment on body weights $(\mathbf{A})$ and soleus weights induced by immobilization in male rats $(\mathbf{B})$. Values are means $\pm S D ; n=6$ animals in each group. \# Significantly different from placebo, $P<0.05$. *Significantly different from contralateral, $P<0.05$. muscle atrophy in the estrogen-treated rat was significantly lower than the placebo group (Fig. 2B). Finally, note that immobilization promoted a loss of total (noncollagenous) protein in both the placebo and estrogen-treated experimental groups. Compared to the placebo animals, however, estrogen treatment significantly retarded the immobilization-induced (percent) loss of total muscle protein in the soleus muscle (Table 1).

$\mathrm{Cu}-\mathrm{Zn}$-SOD protein levels in the estrogen-treated group were significantly lower than in the placebo group. Furthermore, the expression of $\mathrm{Cu}-\mathrm{Zn}-\mathrm{SOD}$ in atrophied SOL from the estrogen-treated group was significantly greater compared with the contralateral one (Fig. 3A). In contrast, no group differences existed in HSP72 levels (Fig. 3B).

In the placebo group, calpain levels increased significantly in the atrophied muscle compared to the contralateral muscle (Fig. 4A). Although cathepsin L protein levels in the atrophied muscle tended to be higher than the con-

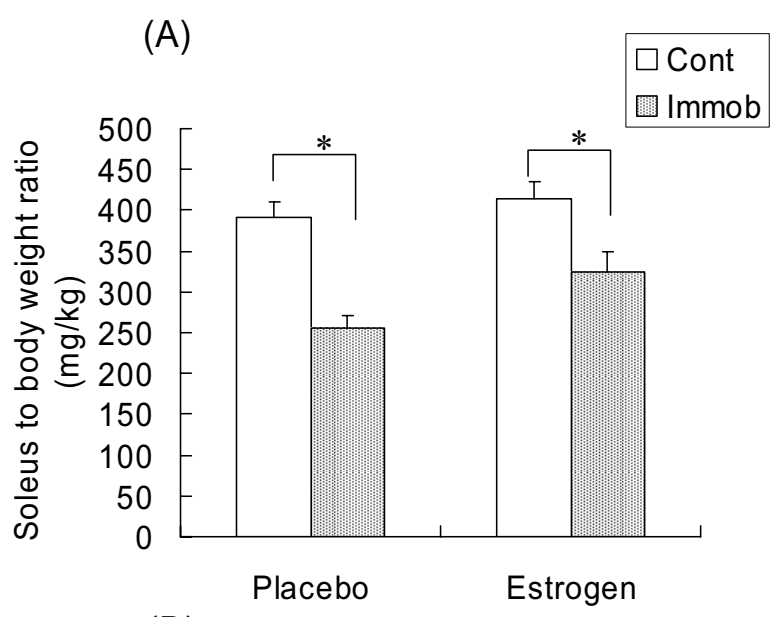

(B)

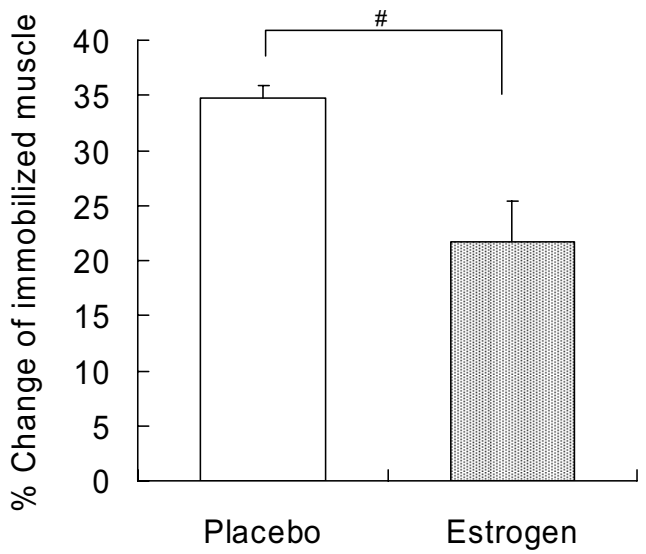

Fig. 2. Effects of estrogen treatment on soleus weight-tobody weight $(\mathbf{A})$ and the degree of atrophy induced by immobilization in male rats (atrophy expressed as percent weight change from contralateral muscle) (B). Values are means \pm $\mathrm{SD} ; n=6$ animals in each group. \# Significantly different from placebo, $P<0.05$. *Significantly different from contralateral, $P$ $<0.05$. 
Table 1. Effects of estrogen treatment on noncollagenous proteins in immobilized soleus muscles.

\begin{tabular}{lccccc}
\hline & \multicolumn{2}{c}{ Placebo } & & \multicolumn{2}{c}{ Estrogen } \\
\cline { 2 - 3 } \cline { 5 - 6 } & Con & Immob & & Con & Immob \\
\hline Total protein concentration $(\mathrm{mg} / \mathrm{g})$ & $208.2 \pm 18.4$ & $175.0 \pm 22.8$ & & $204.2 \pm 12.7$ & $197.9 \pm 31.6$ \\
Total protein content $(\mathrm{mg})$ & $37.1 \pm 5.9$ & $20.6 \pm 5.1^{*}$ & & $32.1 \pm 6.3$ & $24.0 \pm 3.6$ \\
$\quad$ Total protein content expressed as \% of & & $56.5 \pm 9.9^{* *}$ & & $74.9 \pm 7.3$ \\
$\quad$ contralateral muscle & & & \\
\hline
\end{tabular}

Definition of abbreviations: Con, contralateral soleus muscles; Immob, immobilized soleus muscles; g, gram wet weight. Values represent means \pm SD. *Significantly different from contralateral soleus muscles. ** Significantly different from immobilized soleus muscle of estrogen-treated animals.
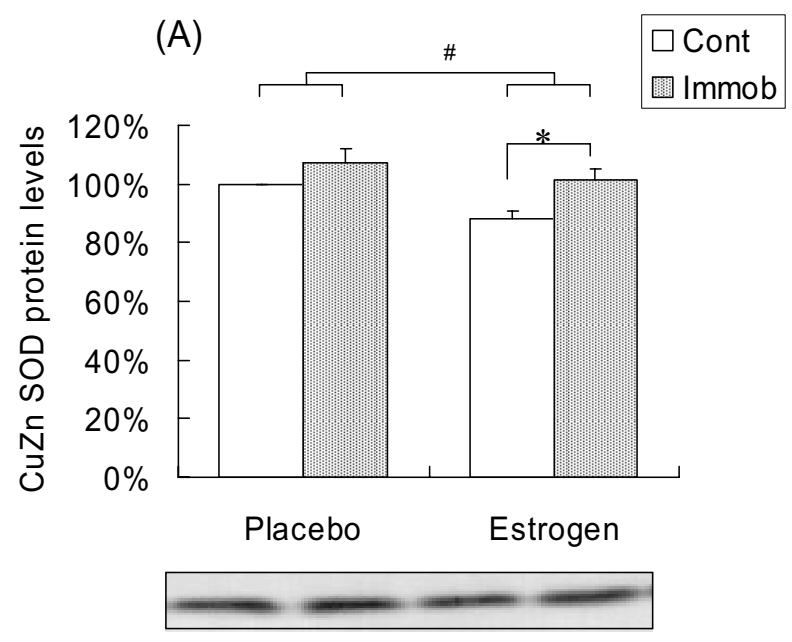

(B)

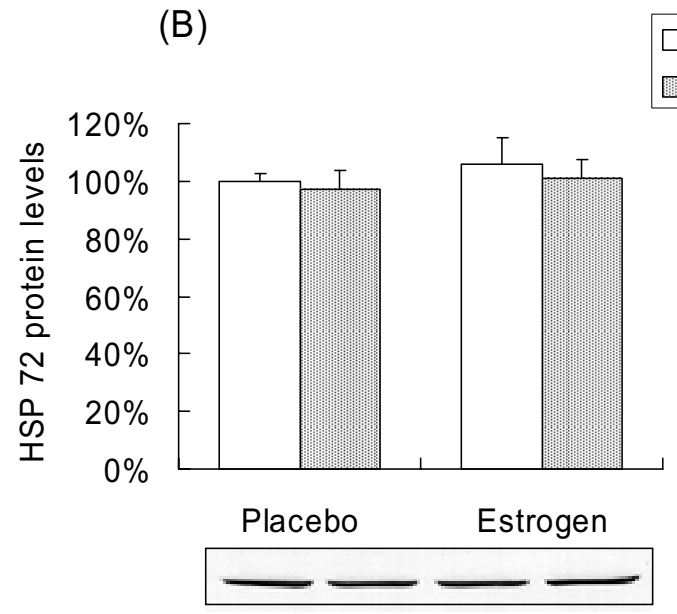

Fig. 3. Effects of estrogen treatment on muscle protein levels of $\mathrm{Cu}, \mathrm{Zn}-\mathrm{SOD}(\mathbf{A})$ and HSP72 (B) in atrophied soleus muscle induced by immobilization of the hindlimbs of male rats. Values are means $\pm S D ; n=6$ animals in each group. \# Significantly different from placebo, $P<0.05$. *Significantly different from contralateral, $P<0.05$.

tralateral muscle, these differences did not reach significance (Fig. 4B). And protein levels of calpain and cathep$\sin \mathrm{L}$ in the estrogen-treated group did not differ between the contralateral and immobilized muscles (Fig. 4, A and B).
(A)

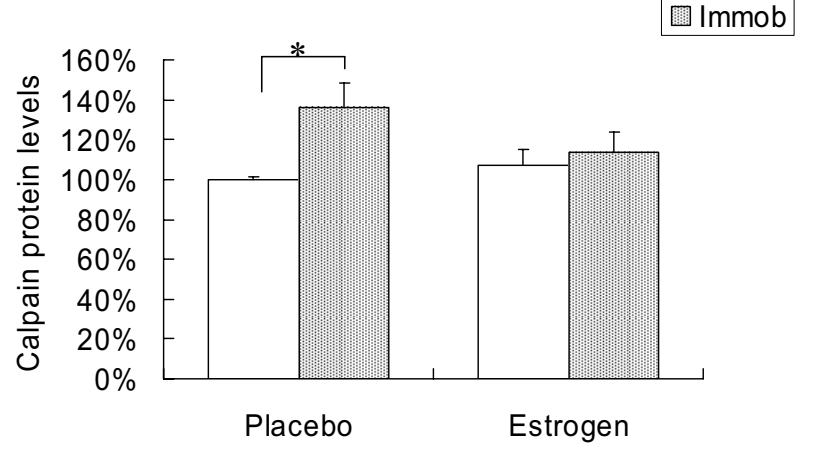

(B)

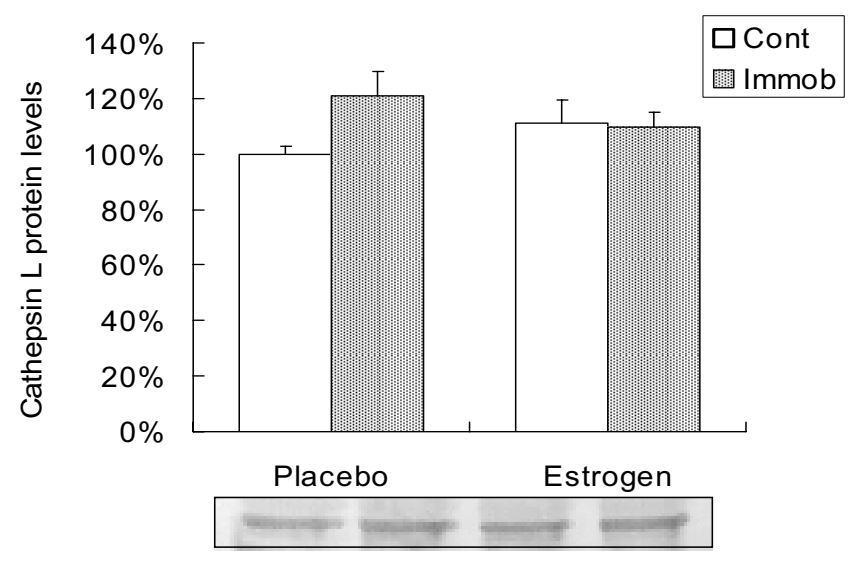

Fig. 4. Effects of estrogen treatment on the expression of calpain (A) and cathepsin L (B) in atrophied soleus muscle induced by the immobilization of hind limbs of male rats. Values are means $\pm \mathrm{SD} ; n=6$ animals in each group. *Significantly different from contralateral, $P<0.05$.

\section{DISCUSSION}

\section{Overview of major findings}

This is the first study to investigate the role of estrogen as a countermeasure to prevent immobilization-induced muscle atrophy. Our data indicate that the delivery of estrogen to male rats retards the rate of muscle atrophy in the immobilized soleus muscle. Moreover, estrogen treatment decreased immobilization-induced increases in muscle 
levels of the calcium-activated protease, calpain. A discussion of these and other key findings follows.

\section{Estrogen and muscle atrophy}

These novel experiments demonstrate that estrogen can retard immobilization-induced skeletal muscle atrophy. It is important to note that the current investigation also provides the first step toward understanding the mechanism(s) responsible for estrogen-mediated protection against disuse muscle atrophy.

It is now well established that prolonged periods of muscular inactivity results in oxidative stress in skeletal muscle fibers, and this redox imbalance accelerates the rate of muscle atrophy $[1,2,4,19]$. Although the precise mechanism by which oxidative stress promotes muscle atrophy continues to be debated, it seems feasible that oxidant damage to muscle contributes to disturbances in cellular $\mathrm{Ca}^{2+}$ homeostasis leading to the activation of calcium-activated proteases such as the calpains and cathepsins [1]. The rationale for this postulate is that the oxidant-mediated formation of reactive aldehydes (i.e., 4hydroxy-2,3-trans-nonenal) reduces plasma membrane $\mathrm{Ca}^{2+}$ ATPase activity [20]. Thus an oxidative stress-induced decline in membrane $\mathrm{Ca}^{2+}$ ATPase activity would diminish $\mathrm{Ca}^{2+}$ removal from the cell, and promote intracellular $\mathrm{Ca}^{2+}$ accumulation. Nevertheless, whether, this mechanism is solely responsible for inactivity-mediated calcium overload in muscle remains unknown.

Our hypothesis that estrogen treatment would retard muscle atrophy in male rats was formulated on the premise that estrogen would act as an antioxidant in the muscle and retard disuse-mediated proteolysis. In this regard, the chemical structure of 17-estradiol includes a phenol ring containing a hydroxyl group with potential antioxidant properties [21]. This feature of estrogen is similar to the chain-breaking action of vitamin E [22]. Similar to vitamin E, experimental evidence demonstrates that estrogen provides physiological protection against radical-mediated lipid peroxidation in rat skeletal muscle $[15,23]$.

Previous work by Kondo et al. [3] indicates that immobilization-induced oxidative stress in skeletal muscle results in an increased expression of $\mathrm{Cu}-\mathrm{Zn}-\mathrm{SOD}$, the cytosolic antioxidant enzyme. $\mathrm{Cu}-\mathrm{Zn}-\mathrm{SOD}$ is a primary cellular antioxidant enzyme and dismutates superoxide radicals to form hydrogen peroxide and water. Our experiments reveal that immobilization promoted an increase in soleus muscle levels of $\mathrm{Cu}-\mathrm{Zn}-\mathrm{SOD}$ in both the placebo and the estrogen treated. Thus from these measurements alone, it is unclear if estrogen attenuated immobilizationinduced oxidative stress in the soleus muscle. However, recent work has shown that the estrogen affects SOD activity and expression. Castrated female rats had lower levels of myocardial SOD activity and serum estradiol concentration compared with sham-operated female rats [24].
Similarly, aortic manganese and extracellular SODs mRNA expressions in ovariectomized mice is lower than that in animals sham-operated and ovariectomized with estrogen supplementation [25]. Assuming that these findings are likely to apply to rat skeletal muscles, we find that estrogen acts as an antioxidant via the stimulation of SOD expression and activity in skeletal muscle.

Recently, Tiidus [26] has reported that calpain activity is diminished in exercised muscles from estrogen-treated animals. The authors suggested that this finding is due to an estrogen-mediated stabilization of the muscle membrane resulting in lower cytosolic levels of free calcium. Moreover, the current data also reveal that estrogen treatment reduced the levels of calpain in immobilized muscles. This is significant because of the important role calpain activation plays in disuse muscle atrophy. It is well established that the bulk of muscle proteins (50$70 \%$ ) exist in actomyosin complexes [27]. Although the proteasome proteolytic system can degrade monomeric contractile proteins (i.e., actin and myosin), this protease does not degrade intact actomyosin complexes [28]. Thus myofilaments must be released from the sarcomere as monomeric proteins prior to degradation by the proteasome system $[27,29]$. This dictates that the release of myofilaments is the rate-limiting step in muscle protein degradation [1]. Since calpain is capable of producing actomyosin disassociation $[27,28,30]$, an activation of calpain is required to achieve proteolytic degradation of myofilaments in muscle during periods of disuse. Therefore our finding that estrogen treatment retards immobilization-induced increases in muscle calpain is consistent with the hypothesis that estrogen treatment retarded immobilization-induced muscle atrophy, at least in part, by an inhibition of protease activation.

\section{Estrogen treatment does not alter muscle levels of HSP72}

Although estrogen promotes the expression of HSP72 in cardiac myocytes [31,32], estrogen treatment in male rats has been shown to prevent exercise-induced HSP72 synthesis in skeletal muscle $[12,33]$. We investigated the combined effect of immobilization and estrogen treatment on the muscle levels of HSP72 because this stress protein may play a protective role in the prevention of muscle atrophy. Based on the collective links between HSP72 and protein synthesis/degradation [34-39], it is possible that elevating HSP72 in skeletal muscle prior to unloading could be a countermeasure to retard disuse-induced muscle atrophy [36]. It follows that a decrease in muscle levels of HSP72 could accelerate the rate of muscle atrophy during periods of disuse. Therefore if estrogen treatment decreased the muscle levels of HSP72, the decline in muscle HSP72 could hasten the rate of muscle atrophy. Nevertheless, our results reveal that estrogen treatment did not alter the soleus muscle levels of HSP72 in the weight- 


\section{T. SUGIURA et al.}

bearing (contralateral control muscle) or immobilized limb. Therefore this finding indicates that an estrogen-induced change in HSP72 is not the mechanism for estrogen-induced protection against disuse muscle atrophy.

It should be noted that Naito et al. [40] reported that the expression of HSP72 was reduced in atrophied soleus muscles induced by hindlimb unloading. Similar results have been reported in denervated skeletal muscles [41]. In contrast, our results reveal that 14 days of muscle inactivity induced by immobilization does not alter muscle HSP72 levels. The discrepancy between our results and previous experiments could be due to differences in the experimental models. For example, in our plaster cast model of muscle immobilization, it is possible for the immobilized muscles to develop muscular tension via isometric contractions. In contrast, muscle contractions do not occur in denervated muscles, and limited force development occurs in muscles subjected to hindlimb unloading. Therefore, although HSP72 levels typically decrease concomitant with decreased muscular activity [40, 41], HSP72 levels in our immobilized muscles did not decrease, perhaps in part, because of periodic isometric contractions of the soleus muscle.

\section{Summary and conclusions}

The current study demonstrates that estrogen treatment attenuates skeletal muscle atrophy induced by immobilization, partly because of the suppression of muscle calpain levels and/or oxidative stress. This is an important finding, and future experiments to delineate the precise mechanism(s) responsible for estrogen-mediated protection against muscle atrophy are warranted.

Currently estrogen therapy is widely used to protect against osteoporosis in postmenopausal women [42]. The present experiments raise the important question, will estrogen therapy also aid in the prevention of age-related sarcopenia? Clearly, further studies are needed to expand our understanding of this important issue, since estrogen treatment has been reported to increase the risk of certain types of cancer [43].

This study was supported in part by a Grant-in-Aid for Scientific Research from Faculty of Education, Yamaguchi University (to T. Sugiura) and the Japanese Ministry of Education, Culture, Sports Science and technology (T. Yoshioka, No. A, 18200042)

\section{REFERENCES}

1. Powers SK, Kavazis A, DeRuisseau K. Mechanisms of disuse muscle atrophy: role of oxidative stress. Am J Physiol Regul Integr Comp Physiol. 2005:288:R337-44.

2. Kondo $\mathrm{H}$, Miura $\mathrm{M}$, Itokawa $\mathrm{Y}$. Oxidative stress in skeletal muscle atrophied by immobilization. Acta Physiol Scand. 1991;142:527-8.

3. Kondo H, Miura M, Itokawa Y. Antioxidant enzyme systems in skeletal muscle atrophied by immobilization. Pflugers Arch. 1993;422:404-6.

4. Betters JL, Criswell DS, Shanely RA, Van Gammeren D, Falk D, DeRuisseau KC, Deering M, Yimlamai T, Powers SK. Trolox Attenuates Mechanical Ventilation-induced Diaphragmatic Dysfunction and Proteolysis. Am J Respir Crit Care Med. 2004;170:1179-84.
5. Ellis S, Nagainis PA. Activity of calcium activated protease in skeletal muscles and its changes in atrophy and stretch. Physiologist. 1984;27 (6 Suppl): S73-4.

6. Taillandier D, Aurousseau E, Meynial-Denis D, Bechet D, Ferrara M, Cottin P, Ducastaing A, Bigard X, Guezennec CY, Schmid HP, et al. Coordinate activation of lysosomal, $\mathrm{Ca}^{2+}$-activated and ATP-ubiquitin-dependent proteinases in the unweighted rat soleus muscle. Biochem J. 1996;316:65-72.

7. Koesterer TJ, Dodd SL, Powers SK. Increased antioxidant capacity does not attenuate muscle atrophy caused by unweighting. J Appl Physiol. 2002;93:195965.

8. Amelink GJ, Kamp HH, Bar PR. Creatine kinase isoenzyme profiles after exercise in the rat: sex-linked differences in leakage of CK-MM. Pflugers Arch. 1988:412:417-21.

9. Bar PR, Amelink GJ, Oldenburg B, Blankenstein MA. Prevention of exerciseinduced muscle membrane damage by oestradiol. Life Sci. 1988;42:2677-81.

10. Dernbach AR, Sherman WM, Simonsen JC, Flowers KM, Lamb DR. No evidence of oxidant stress during high-intensity rowing training. J Appl Physiol. 1993;74:2140-5.

11. Bar PR, Amelink GJ. Protection against muscle damage exerted by oestrogen: hormonal or antioxidant action? Biochem Soc Trans. 1997;25:50-54.

12. Paroo Z, Tiidus PM, Noble EG. Estrogen attenuates HSP 72 expression in acutely exercised male rodents. Eur J Appl Physiol Occup Physiol. 1999;80:1804.

13. Tiidus PM, Bestic NM, Tupling R. Estrogen and gender do not affect fatigue resistance of extensor digitorum longus muscle in rats. Physiol Res. 1999;48:209-13.

14. Tiidus PM. Oestrogen and sex influence on muscle damage and inflammation: evidence from animal models. Curr Opin Clin Nutr Metab Care. 2001;4:509-13.

15. Persky AM, Green PS, Stubley L, Howell CO, Zaulyanov L, Brazeau GA, Simpkins JW. Protective effect of estrogens against oxidative damage to heart and skeletal muscle in vivo and in vitro. Proc Soc Exp Biol Med. 2000;223:59-66.

16. Tiidus PM. Estrogen and gender effects on muscle damage, inflammation, and oxidative stress. Can J Appl Physiol. 2000;25:274-87.

17. Sugita $\mathrm{H}$, Okumura $Y$, Ayai K. Application of a property of troponin to determination of tropomyosin content of a small piece of muscle. J Biochem (Tokyo). 1969;65:971-2.

18. Towbin H, Staehelin T, Gordon J. Electrophoretic transfer of proteins from polyacrylamide gels to nitrocellulose sheets: procedure and some applications. Proc Natl Acad Sci USA. 1979;76:4350-4.

19. Lawler JM, Song W, Demaree SR. Hindlimb unloading increases oxidative stress and disrupts antioxidant capacity in skeletal muscle. Free Radic Biol Med. 2003;35:9-16.

20. Siems W, Capuozzo E, Lucano A, Salerno C, Crifo C. High sensitivity of plasma membrane ion transport ATPases from human neutrophils towards 4-hydroxy2,3-trans-nonenal. Life Sci. 2003;73:2583-90.

21. Subbiah MT, Kessel B, Agrawal M, Rajan R, Abplanalp W, Rymaszewski Z. Antioxidant potential of specific estrogens on lipid peroxidation. J Clin Endocrinol Metab. 1993;77:1095-7

22. Sugioka K, Shimosegawa $Y$, Nakano M. Estrogens as natural antioxidants of membrane phospholipid peroxidation. FEBS Lett. 1987;210:37-9.

23. Stupka N, Tiidus PM. Effects of ovariectomy and estrogen on ischemiareperfusion injury in hindlimbs of female rats. J Appl Physiol. 2001;91:1828-35.

24. Barp J, Araujo AS, Fernandes TR, Rigatto KV, Llesuy S, Bello-Klein A, Singal P. Myocardial antioxidant and oxidative stress changes due to sex hormones. Braz J Med Biol Res. 2002;35:1075-81.

25. Strehlow K, Rotter S, Wassmann S, Adam O, Grohe C, Laufs K, Bohm M, Nickenig G. Modulation of antioxidant enzyme expression and function by estrogen. Circ Res. 2003;93:170-7.

26. Tiidus PM, Holden D, Bombardier E, Zajchowski S, Enns D, Belcastro A. Estrogen effect on post-exercise skeletal muscle neutrophil infiltration and calpain activity. Can J Physiol Pharmacol. 2001;79:400-6.

27. Tidball JG, Spencer MJ. Expression of a calpastatin transgene slows muscle wasting and obviates changes in myosin isoform expression during murine muscle disuse. J Physiol. 2002;545:819-28.

28. Goll DE, Thompson VF, Li H, Wei W, Cong J. The calpain system. Physiol Rev. 2003;83:731-801.

29. Wray CJ, Sun X, Gang GI, Hasselgren PO. Dantrolene downregulates the gene expression and activity of the ubiquitin-proteasome proteolytic pathway in septic skeletal muscle. J Surg Res. 2002;104:82-7.

30. Du J, Wang X, Miereles C, Bailey JL, Debigare R, Zheng B, Price SR, Mitch WE. Activation of caspase- 3 is an initial step triggering accelerated muscle proteolysis in catabolic conditions. J Clin Invest. 2004;113:115-23.

31. Knowlton AA, Sun L. Heat-shock factor-1, steroid hormones, and regulation of 


\section{Estrogen and Muscle Atrophy}

heat-shock protein expression in the heart. Am J Physiol Heart Circ Physiol. 2001;280:H455-64.

32. Voss MR, Stallone JN, Li M, Cornelussen RN, Knuefermann P, Knowlton AA. Gender differences in the expression of heat shock proteins: the effect of estrogen. Am J Physiol Heart Circ Physiol. 2003;285:H687-92.

33. Paroo Z, Dipchand ES, Noble EG. Estrogen attenuates postexercise HSP70 expression in skeletal muscle. Am J Physiol Cell Physiol. 2002;282, C245-51.

34. Thomason DB, Biggs RB, Booth FW. Protein metabolism and beta-myosin heavy-chain mRNA in unweighted soleus muscle. Am J Physiol. 1989;257:R300-5.

35. KuZ, Thomason DB. Soleus muscle nascent polypeptide chain elongation slows protein synthesis rate during non-weight-bearing activity. Am J Physiol. 1994;267:C115-26.

36. Ku Z, Yang J, Menon V, Thomason DB. Decreased polysomal HSP-70 may slow polypeptide elongation during skeletal muscle atrophy. Am J Physiol. 1995;268:C1369-74.

37. Powers SK, Locke M, Demirel HA. Exercise, heat shock proteins, and myocardial protection from I-R injury. Med Sci Sports Exerc. 2001;33:386-92.

38. Smolka MB, Zoppi CC, Alves AA, Silveira LR, Marangoni S, Pereira-Da-Silva L,
Novello JC, Macedo DV. HSP72 as a complementary protection against oxidative stress induced by exercise in the soleus muscle of rats. Am J Physiol Regul Integr Comp Physiol. 2000;279:R1539-45.

39. McArdle A, Dillmann WH, Mestril R, Faulkner JA, Jackson MJ. Overexpression of HSP70 in mouse skeletal muscle protects against muscle damage and agerelated muscle dysfunction. Faseb J. 2004;18:355-7.

40. Naito H, Powers SK, Demirel HA, Sugiura T, Dodd SL, Aoki J. Heat stress attenuates skeletal muscle atrophy in hindlimb-unweighted rats. J Appl Physiol. 2000;88:359-63.

41. Oishi Y, Ishihara A, Talmadge RJ, Ohira Y, Taniguchi K, Matsumoto H, Roy RR, Edgerton VR. Expression of heat shock protein 72 in atrophied rat skeletal muscles. Acta Physiol Scand. 2001;172:123-30.

42. Seeman E, Eisman JA. Treatment of osteoporosis: why, whom, when and how to treat. The single most important consideration is the individual's absolute risk of fracture. Med J Aust. 2004;180:298-303.

43. Weiss NS, Farewall VT, Szekely DR, English DR, Kiviat N. Oestrogens and endometrial cancer: effect of other risk factors on the association. Maturitas. 1980;2:185-90. 